\title{
Las reducciones en Reducciones de Jaime Luis Huenún*
}

\author{
The reservations in Reducciones by Jaime Luis Huenún
}

\author{
Sonia Betancour Sánchez y Orietta Geeregat Vera \\ Universidad de La Frontera, Facultad de Educación, Letras y Comunicación, \\ Departamento de Lenguas, Literatura y Comunicación, Temuco, Chile. \\ Correos electrónicos: sonia.betancour@ufrontera.cl y orietta.geeregat@ufrontera.cl
}

\begin{abstract}
El paradigma occidental perpetuado por la historia oficial de la cultura hegemónica ha significado la condición periférica de la cultura mapuche "subalterna". La obra Reducciones del poeta Huenún, si bien refiere la condición de la cultura "reducida", los significados periféricos implementados por la cultura dominante son resignificados y transformados por multiplicidad de voces a un nuevo estado proveído por una conciencia de mestizaje. Este trabajo observa que la "reducción" impuesta se abre a un nuevo espacio de signos entrecruzados y contradictorios que reformulan el sentido del mestizaje, y discute qué es y cómo es este nuevo espacio, sus significados y modos de textualización.
\end{abstract}

Palabras clave: mapuche, condición mestiza, reducciones, enunciación polifónica

The western paradigm fixed by the hegemonic culture of the official discourse in history has depicted the peripheral condition of the Mapuche subaltern culture. The poetic text "Reducciones" (Reservations) by the poet Huenún if mainly refers to the Mapuche culture "reduced" in the reservations, it also conveys the peripheral constituents given by the dominant culture, which have been transformed and given a new meaning of identity by the many voices provided by the new hybrid status and conscience. The present work watches that the imposed "reservation" opens itself into a new space of woven contradict signs which map and restate the sense of hybrid discussing the way this new place looks like, its actual meaning, and text production.

Key words: mapuche, hibrid condition, reservations, polyphonic discourse

\footnotetext{
Este trabajo forma parte de los Proyectos DI12-0004 "La comunicación intercultural desde el arte mapuche actual", y DI14-0023 "Poetas mapuches. Hacia la configuración de un nuevo imaginario socio étnico: lindes y deslindes de una identidad intelectual autónoma", Universidad de La Frontera, Temuco, Chile.
} 


\section{INTRODUCCIÓN}

La producción artística de los poetas mapuche evidencia un tránsito dinámico de los significados sobre la relación de contacto con la cultura mayoritaria, los que van desde una concepción vertical, hegemónica e históricamente implementada, hasta una concepción horizontal, igualitaria y autónoma. El macrorrelato de esta relación ha visibilizado la cultura minoritaria, instalándola en el espacio público y contribuyendo a un afianzamiento de la identidad cultural. Actualmente, la poesía escrita por personas mapuche mantiene sus rasgos diferenciadores y, al mismo tiempo, algunos de sus textos manifiestan mayor madurez escritural y conciencia intercultural mestiza expresada en los proyectos poéticos de sus creadores. Un ejemplo de esto es el poeta Jaime Luis Huenún en su obra Reducciones.

Reducciones refiere la condición de la cultura "reducida" que incorpora la condición mestiza configurando un nuevo sentido de mestizaje, y la construcción significativa de un nuevo espacio, el de la reducción mestiza, un espacio sin frontera, "el espacio como una articulación significante" (Filinich 2004:70), y donde la significación mestiza es reconstruida a partir de un modo de organización intradiscursivo (Courtés 1997) que traduce la heterogeneidad de la experiencia cultural. En palabras de Bhabha, un "tercer espacio", donde los signos instalados en él "no tienen una unidad o fijeza primordiales" y son "traducidos, rehistorizados y vueltos a leer" (1994: 58-59). A partir de lo anterior, surge la interrogante de qué es y cómo es este nuevo espacio de la reducción mestiza, sus significados y modos de textualización en la obra de Huenún.

Este trabajo postula que la obra Reducciones es un espacio mestizo que refiere la condición de la cultura "reducida", ya no con los significados periféricos implementados por la cultura dominante, sino con los significados propios de un nuevo estado proveído por una conciencia de mestizaje, reelaborando el macrorrelato de la relación cultural a través del desplazamiento del sujeto con una mirada articulada desde los signos de la cultura tradicional.

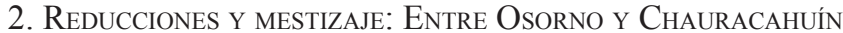

La relación de hegemonía entre mapuche y no mapuche ha sido un tópico permanente en la producción poética mapuche. No obstante, esta historia ha ido transitando hacia historias "otras" en la práctica escritural de los poetas, como dice Mansilla, donde "esta misma historia ha dado paso a la emergencia de nuevas identidades que toman la forma de mestizajes múltiples, dinámicos, subversivos, dolorosos a veces" (2012: 13). El espacio textual es ahora un espacio global de coexistencia de lo indígena y lo no indígena, esto es, un nuevo espacio que aquí llamamos el espacio de la reducción mestiza.

Este espacio es una construcción compleja de entrecruzamientos discursivos, territoriales e identitarios, donde ocurren desmitificaciones culturales (inferior/ superior; nosotros/los otros), destereotipaciones de imaginarios victimizantes (dominador/dominado; despojador/despojado), y discursividades en tensión (oralidad/escritura; textualidad indígena/textualidad occidental). Con estos elementos, entre otros, este nuevo espacio dialoga sin trincheras desde una identidad mestiza que naturaliza el contacto cultural, y que contiene un mundo plural o pluri- 
versal donde no se niega ni se excluye la diferencia. Al contrario, donde distintos mundos generan un encuentro inter-epistémico (mapuche y occidental), con cuyas categorías se resignifica y construye el mundo poético mestizo. De este modo, las categorías indígenas-mapuche hasta ahora subordinadas son activadas e instaladas en el proyecto poético también desde una visión pluri-versal (Mignolo 2010).

El tránsito entre Osorno y Chauracahuín es el desplazamiento entre la reducción impuesta y la reducción reconfigurada desde un sujeto conscientemente mestizo, instalado en un espacio de enunciación contradictorio y ambivalente, un "tercer espacio" (Bhabha 1994) que se abre a la desestabilización de la fijeza de los signos culturales instituidos e investidos de significados excluyentes. Por tanto, hablamos de un espacio global discursivamente reconstruido, intencionado y mestizo.

Reducciones traduce este nuevo espacio global en un espacio donde pierden sentido la etnicidad, la univocidad y la circularidad de los signos predeterminados, porque en el espacio de la enunciación se reconstruyen significados ambiguos, dinámicos, mestizos. Como dice Mansilla, no es "un recuento de tropelías y estropicios cometidos contra los mapuche y mapuche-huilliche a lo largo de cinco siglos (...) poesía reducida: reducida a lamento, a victimización invasora y paralizante de la subjetividad" (2012: 13). Lo anterior subraya la superación de un discurso etnocéntrico e idealista de y sobre la cultura mapuche, que niega su carácter dinámico y muestra el resultado de la natural convivencia y el enriquecimiento entre signos diversos.

El fenómeno descrito permite leer el mestizaje en la obra de Huenún como un espacio-lugar de articulación dialogante de lo indígena, lo no indígena y lo mestizo. Estas diversidades están plasmadas en multiplicidad de voces y textualidades que resemantizan los elementos de la cultura tradicional -la identidad étnico-cultural, la tierra-territorio y los elementos de la cultura impuesta, esto es, el imaginario de la "conquista" y la colonización española, la relación de convivencia y la figura del otro-; todo esto a través de una memoria, ahora mestiza.

De esta manera, se funden, se confunden, dialogan y discrepan en el espaciolugar los variados lenguajes, tiempos y textualidades (peuma, ül, crónicas, cantos funerarios, retrato fotográfico, entre otros), cuya memoria, según Mato (2001), crea una representación que organiza la percepción e interpretación de la experiencia de la historia, reelaborada en la conciencia mestiza, la cual reinstala un saber cultural desestigmatizado y desatado de la racialidad etnocéntrica, favoreciendo una relación intercultural horizontal. La representación de esta memoria en Huenún incorpora la vivencia con el otro, como un aspecto positivo que favorece el desarrollo cultural y fortalece los saberes de cada quien.

La memoria está en función de mantener la identidad cultural, la que al instalarse en el espacio de contacto reescribe el pasado de manera selectiva, y enfatiza en el presente los elementos culturales que definen lo propio en la mezcla con lo ajeno. En este mismo sentido citamos las palabras del poeta mapuche Bernardo Colipán, quien sostiene que el huilliche "se encuentra interiormente cruzado por distintos tiempos -el cotidiano y el de la memoria- que se integran y se contradicen en una permanente dialéctica en suspenso, que no es inoportuna y que sólo a ratos incomoda" (Cit. en Fierro y Geeregat 2002: en línea). Esta apreciación expresa ya la complejidad del tiempo mapuche, la cual se profundiza aún más en el tiempo presente del espaciolugar mestizo, como resultado del difícil tránsito entre Osorno y Chauracahuín. 
El desplazamiento simbólico entre Osorno y Chauracahuín trae a la memoria la contradicción de los sucesos, donde, por una parte, los elementos culturales aparecen complementándose en la Plaza de Armas de la ciudad de Osorno: "Mediaba el mes de mayo de 1938 cuando la sociedad osornina rindió tributo blanco a aquella mujer morena (...) a pesar del color diaguita de su piel y del cielo aymara prendido en sus verdes pupilas de Vicuña"; y, por otra, están los hechos de Forrahue, los misioneros capuchinos bávaros, la llegada de los migrantes alemanes a Chauracahuín que junto con los colonos chilenos se apoderaron de "terrenos indios", la instauración de la "Ley de Colonización" del año 1851, todos hechos que son "todavía una cicatriz en la memoria de los viejos huilliche de San Juan de la Costa” (Huenún 2012: 23-25). La reducción originada a partir de esta ley transformó simbólicamente a Chauracahuín en Osorno, donde:

Ahora en las grandes praderas de los fundos osorninos pastan las vacas Holstein y los rojos toros Hereford (...). Muchas familias huilliche convertidas al catolicismo entregaban sus hijos a las Misiones religiosas apostadas en lugares estratégicos del otrora territorio indígena (...). En este proceso civilizatorio y cristianizante se cortaba de raíz el cordón umbilical de la lengua che sungun, y se adiestraba a los alumnos en labores domésticas y agrarias con el objetivo de integrarlos al sistema económico vigente (Huenún 2012: 23-25).

Esta complejidad histórica es revisada desde la mirada actual del sujeto poético como un resabio dinamizador capaz de construir un espacio sociocultural translocado, cuyas ambivalencias y contradicciones concurren en una identidad cultural mestiza. La complejidad de estos conceptos -identidad-mestizaje- parte de su condición de categorías predeterminadas por la cultura dominante, con las cuales se observa a la cultura dominada desde una mirada parcializada, polarizada y dual que no concibe la mezcla y, por tanto, no la deja ver:

las aproximaciones dualistas y maniqueas seducen por su simplicidad (...). Podemos reducir así la historia de la conquista de América a un enfrentamiento destructor entre buenos indios y malos europeos (...). Esta manera de ver las cosas petrifica y empobrece la realidad (...) los intercambios entre un mundo y otro, los cruces (...) (Gruzinski 2000: 48).

Siguiendo las ideas de este autor, pensar la mezcla requiere pensar también el uso simplista del término cultura, imbuido de una pureza romántica y falaz que niega la mezcla y bloquea la porosidad de las fronteras, y con el cual es imposible pensar que "un buen hombre es un hombre mezclado"(2000: 49-52). Este modo de concebir las culturas lleva a la permanente reiteración de un nosotros/otros como se observa, por ejemplo, en la relación dominador (superior)/dominado (inferior) en la historia de América, a través de los términos: europeo-conquistador/indígenaconquistado, y en la historia de Chile, a través de los términos español-conquistador/ mapuche-conquistado, fenómeno que en el caso chileno se vuelve a reiterar en la relación intercultural conflictuada chileno-mapuche, a través de los términos: pueblo mapuche-usurpado/Estado chileno-usurpador, mapuche-víctima/wingka-victimario.

Como observamos en estos términos, el estereotipo indígena y europeo se construye sobre representaciones binarias. Una observación que nos parece 
interesante es que si bien a los indígenas o mapuche en Chile les han sido atribuidos los calificativos de "indio, flojo y borracho" (Saiz 1986:123), también es cierto que la categoría del estereotipo ha sido incorporada por los indígenas hacia lo chilenooccidental, calificándolo de wingka/usurpador, abusador, ladrón, entre otros, cuestión que da cuenta de una forma maniqueísta de pensamiento. Estas categorizaciones, independientemente de quienes las establezcan, se reducen a la categoría macrosemántica positivo v/s negativo, que se expresa en nosotros-buenos/los otrosmalos.

En la obra de Huenún esta categoría no está ausente, su estructuración discursiva palimpséstica de intencionado entrecruzamiento y aspiración dialógica desdibuja los pares opuestos y sus significados excluyentes, reuniéndolos en una voz con sentido polifónico y de reducciones socioétnicas. Consecuentemente, acordamos que en el ser humano habitan múltiples identidades, las cuales se activan dependiendo de los contextos, y sólo se pueden definir en función de las múltiples relaciones e interacciones en que se manifiestan. Entonces, la relación nosotros/otros se vuelve estéril y no da lugar al espacio intersticial (Bhabha 1994) donde se produce la complejidad de la relación humana, intersticios donde Huenún articula los elementos culturales seleccionados y construye nuevos significados sobre la reducción.

En este espacio surge el mestizaje perturbando el orden de lo predecible, de los acontecimientos y del devenir histórico previsto, un rompimiento de lo lineal que hace confluir tiempos y espacios sin una devastación, sino en un proceso dinámico de movimiento pendular entre el orden y el des-orden, esto es, al decir de Gruzinski, "el modelo de la nube", "una forma desesperadamente compleja, vaga, cambiante, fluctuante y siempre en movimiento. Los mestizajes pertenecen a este orden de realidad" (2000: 60), concepción a la que se adscribe este trabajo.

\section{ESTRATEGIAS DISCURSIVO-COMUNICATIVAS}

En este proyecto poético se observan estrategias discursivo-comunicativas que resignifican la reducción de la relación intercultural, traduciéndola a sus significados mestizos o, al menos, abriéndose a esta posibilidad. Es decir, una relación intercultural no reducida al dualismo buenos/malos sino, como ya se señaló, a una condición compleja que desdibuja esta reducción.

La obra de Huenún es un texto vital e inacabado, que se va (entre) tejiendo en torno a la macrocategoría reducciones, la cual se articula a través de diferentes voces, significados y textualidades, cuyos niveles -nivel del sujeto, nivel semántico y nivel superestructural- (García, Betancour y Geeregat, en revisión) potencian esta condición del texto, el cual, en palabras de Lotman, "ya no es más un portador pasivo de significado, sino que aparece como un fenómeno dinámico e intrínsecamente contradictorio" (Cit. en Gaínza 2010: en línea). En este tejido el sujeto de la enunciación construye el mundo de la obra con un fuerte grado de conciencia sobre su ser mapuche mestizo, lo que se evidencia en su condición de arraigo étnico cultural, re-creada en el devenir histórico del sujeto.

Los sucesos históricos sobrevinientes en este nuevo estado se hacen parte del yo interno, vinculándose en él de manera profunda y contradictoria, en un encuentro entre los signos profanos de la irrupción y los signos sacros del arraigo. Algunos ejemplos de esta condición son: 
- Explicitación de aspectos biográficos, su linaje: "provengo, por sangre paterna, de un tronco huilliche..." (p. 99).

- Prácticas culturales, cotidianas: "junto al río de estos cielos / verdinegro hacia la costa, / levantamos la casa de Zulema Huaiquipán" (p. 131); "Yo la miro / danza / canelo florecido lleva en sus manos / danza / (...) / ríe y danza / bebe su muday" (p. 63).

- Prácticas culturales, rituales: "Regresa el sol a la tierra, / a los ríos y a los árboles / y a las semillas sembradas / en los cerros y en los valles (...) / ¡We Tripantu, We Tripantu! / dicen los ancianos padres, / cantándole al nuevo sol / que en sus corazones arde" (p. 144).

- Actos de irrupción: "Así vinieron ellos, con hachas y cuchillas, / derribando solares, púlpitos y alcobas. / ¿Habrá visto, Usía, las púberes mancebas / aullando sobre el lomo de los indianos lóbregos?” (p.32); “(...) nos hablaron bajito y nos dieron garrotes, / y unos tragos de pisco para aguantar el frío. / Nadamos muy ligeros para no acalambrarnos. / La neblina cerraba la vista de la orilla. (...) / nos marchamos borrachos, emplumados de muerte, / cantando unas rancheras y orinando en el viento" (p. 113).

- Sentimientos de intemperie y desarraigo: "Aquí, henos aquí, / ya viudos de nuestros dioses, viudos del sol, de agua / y de la luna llena / (...) / ¿Para quién brilla el laurel? / ¿Para quién moja sus ramas? / De lejos se escucha el mar, / y el graznido del güairao" (p. 112).

- Muerte trágica y transformación heroica: “De dónde viene el hilo de una larga mirada? / ¿Y el color de la muerte en las flores del mar? (...) / Huesos que resuenan, lunas que circulan / sobre niños huyendo de tábanos azules. (...) / La muerte casi al alba arde en las cordilleras, / la luz, como una herida, rompe el ventanal" (p.155).

La instancia de la enunciación evidencia un sujeto-yo que generalmente se funde en un nosotros inclusivo, conocedor de la historia y de sus desplazamientos, catalizador de los sentimientos humanos, de la naturaleza en sus pálpitos vegetales y animales. Es un sujeto que, al situarse en la esfera de la cultura, funde el tiempo mítico con el tiempo actual, como en los versos: "El sol y el mar harán rulamas / que sacaremos de la roca. / (...) / Huenteao habla en cada ola, / y con sus nubes tapa el sol". Apela a un tú, instruyéndole en las prácticas culturales rituales: "no mires mal, hermana, no, / no mires mal hacia la Isla / (...) / Báilale bueno un cielito, / tócale banjo y mandolina" (p. 60).

Al mismo tiempo, instruye a un destinatario con competencia cultural relativa a través de la inclusión de un texto complementario explicativo en la parte inferior de la página, para que comprenda los lenguajes y significados de la cultura tradicional, en este caso el ritual de Huenteao: "Los viejos huilliche de la provincia de Osorno aún realizan el viaje ritual y alimenticio hasta las playas de Pucatrihue (...)" (p. 60).

El sujeto cuenta y re-construye el universo poético desde la profundidad y la contradicción de sus sentimientos, en un tiempo lineal donde se sitúan los hechos de la irrupción -espacio-tiempo pretérito-, y en un tiempo mítico -espacio-tiempo circular-, cuyos deícticos y "subjetivemas" (Filinich 2004: 41-42) contribuyen en ambos tiempos con significados de muerte y de vida; de destrucción y de reconstrucción; de identidad cultural propia y de identidad cultural impuesta.

Las valoraciones del sujeto sobre el mundo que enuncia crean un clima intencionado que busca la complicidad del destinatario y lo obliga a articular los significados propuestos de aquél con los de éste, en un ejercicio colaborativo y 
fraterno: "Sin embargo, la memoria de otros tiempos todavía alumbra a los más de 50.000 huilliche que habitan las reducciones de San Juan de la Costa (...). Y aunque la lengua originaria sólo sobreviva en un puñado de ancianos, quedan todavía ceremonias a que convocan comuneros de diversos credos y linajes" (p. 26).

Las variadas voces que hablan desde la multivocidad y el empleo de distintas formas textuales, crónicas, testimonios, canto ancestral, cartas, entre otras, funcionan como potentes documentos al servicio del sentido de totalidad buscado por el sujeto, y orientado por la insistencia de la pregunta retórica, la reminiscencia y la voz imperativa: “¿Para quién brilla el laurel? / ¿Para quién moja sus ramas? / (...) / Nadie / me responde, nadie. Sólo / estoy ante la noche” (p. 112); “¿Quién hará oraciones a la tierra, / al ciruelo, a la higuera, al boldo tibio, / quién dará primavera a las raíces / y descanso al cielo y a los árboles?” (p. 137); “(...) / Mirad la enredadera cubriendo los umbrales / de viejos caserones donde ladran los perros, / mirad las blancas sombras en las puertas vencidas / de una larga ciudad enfrentada a los astros" (p. 49).

Se trata de una voz que refiere lo sacralizado y lo profano, lo urbano y lo rural, lo sublime y lo cotidiano, en universos fragmentarios que, en su conjunto, son una sinécdoque del mestizaje, una totalidad pluriversa, contenedora de un lugar nuevo y viejo a la vez, mutable y perenne, donde el sujeto se desplaza en su ser indígena por un espacio "otro" que lo envuelve y lo desafía a enfrentarse a su inevitable mutabilidad. En este sentido, el espacio urbano es una amenaza a la cultura ancestral, pero al mismo tiempo la reafirma en los signos culturales del resguardo y en los signos de la apertura: "Abandoné mi casa / abandoné mi luz / esa carcacha matutina / que indicaba / un camino a la belleza / Oye tú / díjome la anciana / chupa / mi teta huilliche / sorbe su sabor a piedra negra / lame su fuego de huesos y pelambres / Y yo lamí / bebí / sorbí / mordí / y luego me retiré a mis aposentos / de la Gran Avenida / (...)” (p. 158).

El espacio de contacto cultural, inevitablemente, termina produciendo un encuentro entre los elementos sacros de la cultura propia y los elementos cotidianos de la cultura ajena; la "teta huilliche" es la condición umbilical del sujeto con el espacio materno, nutricio, inoculador de identidad, metáfora de la mapu ñuke, protegido por Nguenechén, cuya investidura divina es la misma de la divinidad occidental. La convivencia cotidiana, por su parte, reelabora los sentidos mestizos en la práctica escolar occidental, donde los elementos se desplazan dinámicamente entre lo mapuche y lo no mapuche, desdibujando límites, ampliando el espacio de la reducción:

(...) los ojos y los oídos de Nguenechén, la divinidad mapuche que después de 120 años de derrota aún pervive confundida entre el credo católico y el protestante. Los niños, en tanto, acuden a la escuela a escribir el sol en castellano. En medio del camino escuchan el canto del chucao y contemplan, silenciosos, el vuelo del cernícalo de monte. Mañana, sin embargo, viajarán a la ciudad. Hablarán allá de los abuelos que contaban, sin apuro, sus visiones y sus sueños frente al fuego y la neblina del amanecer (p. 141).

La polifonía enunciativa es entendida como la circulación de otras voces en el discurso propio, a través de las cuales incorpora ideologías, creencias, valores (Filinich 2004: 46). En este caso, valiéndose fundamentalmente de los textos cronísticos, históricos y visuales, incorpora voces y acontecimientos pretéritos que son transformados en otros textos, un fenómeno de "mutación disciplinaria" según Iván Carrasco (2003), y que son actualizados y resignificados en la obra poética. 
Un ejemplo de esto es, entre otros, el texto Sermón en lengua de Chile (p. 36) del misionero jesuita español Luis de Valdivia, un texto histórico del año 1621 que refiere el proceso de evangelización como recurso de solución del entonces problema de resistencia mapuche, donde la voz poética se desdobla en otra voz que, al tomar su lugar, extiende y tensiona los significados del discurso oficial en el discurso poético. Esto, en palabras de Yahalom, constituiría “el fenómeno de la interacción recíproca entre el texto literario que se disfraza como no literario y el texto no literario que le sirve de referencia -interacción que contribuye a la formación del campo literario-" (Cit. en Iglesias 1999: 110).

En este mismo sentido, el despliegue simultáneo de los elementos de los textos visuales de "Cuatro cantos funerarios" (Huenún 2012: 70-75) delimita los significados del discurso histórico. Intenciona así de modo subyacente una percepción crítica de la dimensión humana como valor universal, arrasado por una práctica cultural ajena.

El significado del "museo", por ejemplo, se expone en el sentido original de espacio reducido, como un reservorio histórico de significados fosilizados. No obstante, la fidelidad de los textos está al servicio de una provocación, lo menos que se puede pensar es ¿qué se preserva en un museo?; lo exótico, lo que ya no está, el testimonio de una existencia pasada, un objeto de estudio, de admiración, de exhibición, de interés colectivo, entre otras posibilidades. La función descriptivoexplicativa de los textos verbales invisibiliza la dimensión humana del sujeto-objeto reducido y la violencia de los sucesos referidos.

Los textos visuales, por su parte, confirman y amplían los significados literales y los datos objetivos de modo tan evidente y revelador que lo natural en el museo se lee como una aberración, un contra sentido que, en lugar de refrendar la historia oficial, la reescribe con significados obscenos. Se trata de una historia que se revierte en un discurso poético de profundo lamento, una elegía en cuatro cantos que, a partir de esta condición, sale del reducto del "museo" a otro espacio-tiempo más amplio y visible para miradas otras, el de las "reducciones" del discurso poético, que entrega múltiples posibilidades de sentido, siendo, tal vez, éste el intencionado propósito del proyecto poético de Huenún: resarcir el daño del suceso y del discurso histórico institucionalizado.

En "Cisne de mi" la enunciación cede la voz a la figura del cisne que replica en versos la historia, se convierte en la metáfora del dolor: "Cisne de mí, negrura de mi cuello / que oculto bajo el cielo de las aguas turbias, / hundido el corazón, perdido el canto, / lejana la bandada, de mi sangre/ sangro" /... / (p. 74). La soledad inmensa que lo envuelve le ofrece, a su vez, la compañía cómplice de la naturaleza como refugio al espíritu atribulado, un lugar de remanso, de redención. Desde aquí, la voz desplaza los significados de la desolación de un tiempo finito y ajeno hacia un lugar-tiempo propicio, perenne y trascendente. El cisne comporta así todo su valor simbólico de pureza, blancura excelsa, intocado, capaz de sacrificarse por todos, en una condición mesiánica y sublime.

El carácter polifónico del texto, junto con incluir variables textuales y mutación disciplinaria, también incorpora voces a través del diálogo entre otros hablantes, cuya forma lingüística castiza, "E fablan lingüa bárbara", por ejemplo, informa y califica a una lengua diferente, "bárbara", rústica, y un obstáculo para la evangelización, como se observa en el poema Che Sungun (p. 33).

Los textos con sentido irónico -otra dimensión de la polifonía- constituyen una crítica al vasallaje cultural de occidente, fenómeno reiterado en la mayoría de 
los textos de "Entrada a Chauracahuín". En ellos la voz en su expresión irónica revierte y desplaza los significados históricos a textos poéticos, cuya estructuración intradiscursiva contribuye a una ambigüedad semántica sobre la acción colonizadora (idioma, religión) y el sistema creencial mapuche.

Otros recursos polifónicos que favorecen los desplazamientos de sentido son epígrafes, citas, glosarios y notas al pie, los cuales, al recontextualizar los sucesos, dialogan con los textos poéticos afectando los significados. Y donde la voz enunciativa propone lecturas y valoraciones que no sólo reclaman la competencia intercultural del lector, sino que le comprometen emocionalmente y/o extienden su conocimiento.

Es el caso, por ejemplo, de los poemas que aluden a la muerte de Matías Catrileo (Ül de Catrileo, p. 156) y Jaime Mendoza (Jaime Mendoza Collío se pierde y canta en los bosques invisibles de Requém Pillán, p. 155), o el epígrafe del texto Uno Forrahue (p. 104), entre otros. Estos desplazamientos de sentido originados por los recursos polifónicos nutren el nuevo espacio, el espacio de "reducciones", metáfora del encuentro y desencuentro interétnico e intercultural, sintetizado y revitalizado en los significados mestizos.

Retomando la condición dinámica y contradictoria del tejido textual ya señalada, subrayamos los significados inestables como principio constitutivo de lo mestizo, sustentado en lo indígena-ancestral, conciencia de un sujeto inserto en un espacio de signos culturales mezclados en la visión y/o anhelo de un espacio materno -la madre tierra/mapu ñuke- como fuerza superior que lo conmina a volver a nutrirse de su raíz, de "su teta huilliche":

(...) Oye tú / díjome la anciana / chupa / mi teta huilliche / Sorbe su sabor a piedra negra / lame su fuego de huesos y pelambre / Y yo lamí / bebí / sorbí / mordí / Y luego me retiré a mis aposentos / de la Gran Avenida / Me tendí después sobre la bandera de Chile / dejé caer mi nuca sobre la estrella iluminada / y soñé despierto: / Esto debo esto / no debo comer / esto es / del Fondo Monetario Internacional: / (...) (Carta de los suelos, p. 158).

La condición mestiza se reafirma también en la hibridez de los textos y las relaciones intertextuales propuestas. Cabe destacar el texto "Lucho Llanquilef envía su última carta desde el Río de la Greda" (p. 151), cuyo cuerpo textual es un discurso de la memoria (Fierro 2005) en forma de carta abierta que un Yo envía a un Tú in extrema res, quien, desde el día de su funeral, relata su vida anterior.

Este texto constituye un verdadero collage intertextual sustentado en un símil con La Amortajada de María Luisa Bombal, donde, al modo de Ana María, el sujeto de la enunciación, desde su condición de amortajado dentro del ataúd "de pino", exterioriza su yo interior "mientras el cortejo avanza bajo el cielo que se abre y se ilumina para mi". En este fluir de la conciencia la experiencia de la muerte lo conecta con la experiencia de la vida, donde el ritual de despedida incorpora las canciones de "Los Reales del Valle"; su reminiscencia incluye el parafraseo de la letra de una conocida canción chilota "Fui pescador y lobero"; un dicho popular chileno "pata de cumbia" que alude a la cojera de una persona, y; películas de cine "Los siete magníficos, La muerte tenía un precio y Por un puñado de dólares".

Estas relaciones intertextuales evidencian la condición mestiza -no sacralizadavivenciada por el sujeto, y un imaginario igualmente mestizo corroborado por 
lugares citadinos y acciones culturales no mapuche: el "camposanto de la Misión de Quilacahuín, la tienda Embajadores, el cine, las quintas de recreo de Osorno, de La Unión y Río Bueno, las boîtes, la plaza de Punta Arenas, el Gran Santiago"; destaca el funeral y los signos cristiano-occidentales que concurren en él: "el suelo de Dios, el campo santo, la sábana de Verónica, rostro cubierto, el ataúd de pino, pobre velorio".

El traslado en "un bote hasta el suelo de Dios" en la cultura mapuche es el viaje ritual de la muerte, que se entiende como el tránsito con la ayuda del balsero, desde una dimensión de la existencia a otra. No obstante, esta acción también se presenta como ritual cristiano-occidental en la forma literal de traslado en bote de un ataúd en invierno, en la zona de San Juan de la Costa.

La reiteración "Aún no muero, sobrino", que abre y cierra el texto epistolar, expresa más de una posibilidad de sentido, la posibilidad literaria de relacionar al sujeto de la narración tanto con el anhelo de trascendencia cristiano-occidental como con la manera de la cosmovisión mapuche, el viaje, un estado intermedio imposible de nombrar: "Cuál es mi nombre ahora sobre el agua brusca del invierno" que representa el cruce del río del cuerpo ya sin hálito, pero que no muere en lo espiritual; un viaje físico al cementerio de la "Misión de Chauracahuín".

Este espacio reducido de la morada terrenal se ha transformado en un espacio mestizo. En él concurren la dimensión simbólica mapuche y la dimensión material occidental, ampliando el sentido de la reducción desde un signo occidental de finitud, hacia un signo resemantizado de la permanencia del espíritu mapuche en la naturaleza, donde termina el viaje de la memoria y se re-inicia el sentido circular de la existencia.

\section{Conclusiones}

La obra Reducciones es un espacio global, discursivamente reconstruido, donde dialogan los signos de la contradicción y la ambivalencia constitutivos de lo mestizo. Como se señaló, es un espacio-lugar de discursividades complejas, espacio en el cual los elementos culturales del contacto desplazan los significados unívocos y excluyentes, dando lugar a significados múltiples y porosos por medio de una polifonía discursiva.

En este espacio-lugar el mestizaje produce un movimiento dinámico, pendular, constante, que coincide con una forma compleja y fluctuante (Gruzinski 2000) de la realidad mestiza. Este tercer espacio (Bhabha 1994) -espacio entre Osorno y Chauracahuín- es el tránsito entre la reducción impuesta y la reducción reconfigurada por una enunciación ambivalente que desestabiliza los elementos culturales asignados, ajenos y localizados, e instala este espacio global de las reducciones mestizas, donde el sujeto en el contacto cultural elabora un acto dialógico al modo de un zugatrawun en un discurso de la memoria (Fierro y Geeregat 2004), una memoria híbrida que entrecruza territorios e identidades, desbordando límites y reducciones.

Las estrategias discursivas, polifonía de voces, textualidades múltiples, mutación disciplinaria, ironía, sinécdoque, enunciación ambivalente y memoria, sirven, fundamentalmente, al propósito de la reescritura de las reducciones como metáfora de identidad mestiza. En esta obra el poeta Huenún reafirma la coherencia de su postura y discurso mestizo, condición vinculada a la honestidad con la cual reconoce, incorpora y valida elementos culturales mapuche y no mapuche, como aporte en la construcción de su discurso poético. 


\section{OBRAS CITADAS}

Bhabha, Homi. 1994. El lugar de la cultura. Buenos Aires: Manantial.

Carrasco, Iván. 2003. "La antropología poética como mutación disciplinaria". Estudios Filológicos 38: 7-17. Recuperado el 17 de julio de 2013, de http://www.scielo.cl/scielo. php?script=sci_arttext\&pid=S0071-17132003003800001\&lng=es\&tlng=es. $\quad 10.4067 /$ S0071-17132003003800001

Courtés, Joseph. 1997. Análisis semiótico del discurso. Del enunciado a la enunciación. Madrid: Gredos.

Fierro, Juan M. y Orietta Geeregat. 2002. "Testimonios poéticos del mestizaje mapuche. Memoria y contramemoria en textos de Elicura Chihuailaf, Leonel Lienlaf, Jaime Huenún y Bernardo Colipán". Razón y Palabra 26. Recuperado el 17 de julio de 2013, de http:// www.razonypalabra.org.mx/anteriores/n26/jmfierro.html

2004. "La memoria de la Madre Tierra: El canto ecológico de los poetas mapuches". Anales de Literatura Hispanoamericana 33: 77-84.

2005. El discurso de la memoria en Chile (1970-2005). Tesis Doctoral. Valdivia: Universidad Austral de Chile.

Filinich, Ma Isabel. 2004. Enunciación. Buenos Aires: Eudeba.

Gaínza, Gastón. 2010. "Pespuntes Semióticos II". Entretextos. Revista Electrónica Semestral de Estudios Semióticos de la Cultura 14-15-16. Recuperado el 17 de julio de 2013, de http://www.ugr.es/ mcaceres/entretextos/entre14-16/gainza.html

García, Mabel, Sonia Betancour y Orietta Geeregat. En revisión. "Estrategias comunicativas del Pueblo Mapuche en el espacio de contacto cultural". Enviado a Convergencia. Revista de Ciencias Sociales.

Huenún, Jaime. 2012. Reducciones. Santiago de Chile: LOM Ediciones.

Mansilla, Sergio. 2012. "Los archivos de la niebla (notas para leer Reducciones de Jaime Luis Huenún)”. En Huenún, Jaime Luis, Reducciones. Santiago de Chile: LOM Ediciones. 11-20.

Mignolo, Walter. 2010. Desobediencia epistémica: Retórica de la modernidad, lógica de la colonialidad, gramática de la descolonialidad. Buenos Aires: Ediciones del Signo.

Saiz, José. 1986. "Estereotipos adscritos al indígena Mapuche por adultos no Mapuches de Chile Meridional”. Revista Interamericana de Psicología 20.1/2: 55-58.

Gruzinski, Serge. 2000. El pensamiento mestizo. Barcelona: Paidós Ibérica.

Mato, Daniel. 2001. "Producción transnacional de representaciones sociales y cambio social en tiempos de globalización". En Mato, Daniel (Coord.), Estudios latinoamericanos sobre cultura y transformaciones sociales en tiempos de globalización. Buenos Aires: CLACSO. 127-160. 
